\title{
Feedback Control Variables Have No Influence on the Permanence of a Discrete $n$-Species Schoener Competition System with Time Delays
}

\author{
Qianqian Su and Na Zhang \\ College of Mathematics and Computer Science, Fuzhou University, Fuzhou, Fujian 350108, China \\ Correspondence should be addressed to Qianqian Su, suqian112@yahoo.cn \\ Received 1 May 2010; Accepted 15 July 2010 \\ Academic Editor: Antonia Vecchio
}

Copyright (c) 2010 Q. Su and N. Zhang. This is an open access article distributed under the Creative Commons Attribution License, which permits unrestricted use, distribution, and reproduction in any medium, provided the original work is properly cited.

We consider a discrete $n$-species Schoener competition system with time delays and feedback controls. By using difference inequality theory, a set of conditions which guarantee the permanence of system is obtained. The results indicate that feedback control variables have no influence on the persistent property of the system. Numerical simulations show the feasibility of our results.

\section{Introduction}

The Schoener's competition system has been studied by many scholars. Topics such as existence, uniqueness, and global attractivity of positive periodic solutions of the system were extensively investigated and many excellent results have been derived [1-5].

Liu et al. [1] propose and study the global stability of the following continuous Schoener's competition model with delays:

$$
\begin{aligned}
& \dot{x}_{1}(t)=x_{1}(t)\left[\frac{a_{10}(t)}{x_{1}\left(t-\tau_{10}\right)+k_{1}(t)}-a_{11}(t) x_{1}\left(t-\tau_{11}\right)-a_{12}(t) x_{2}\left(t-\tau_{12}\right)-c_{1}(t)\right], \\
& \dot{x}_{2}(t)=x_{2}(t)\left[\frac{a_{20}(t)}{x_{2}\left(t-\tau_{20}\right)+k_{2}(t)}-a_{21}(t) x_{1}\left(t-\tau_{21}\right)-a_{22}(t) x_{2}\left(t-\tau_{22}\right)-c_{2}(t)\right],
\end{aligned}
$$

where $\left\{k_{i}(t)\right\},\left\{a_{i j}(t)\right\}$, and $\left\{c_{i}(t)\right\}$ are all positive bounded and continuous functions, $\tau_{i j}(i=$ $1,2 ; j=0,1,2)$ are positive integers, $\tau=\max _{0 \leq i, j \leq 2}\left\{\tau_{i j}\right\}, x_{i}(s)=\phi_{i}(s) \geq 0, s \in[-\tau, 0] \cap Z$, $\phi_{i}(0)>0, i=1,2$. 
As we all know, though most dynamic behaviors of population models are based on the continuous models governed by differential equations, the discrete time models are more appropriate than the continuous ones when the size of the population is rarely small or the population has nonoverlapping generations. It has been found that the dynamics behaviors of the discrete system are rather complex and contain more rich dynamics than the continuous ones [6]. Recently, more and more scholars paid attention to study the discrete population models (see [4-13] and the references cited therein). For example, [5] considered the permanence and global attractivity of the following discrete Schoener's competition model with delays:

$$
\begin{aligned}
& x_{1}(n+1)=x_{1}(n) \exp \left\{\frac{a_{10}(n)}{x_{1}\left(n-\tau_{10}\right)+k_{1}(n)}-a_{11}(n) x_{1}\left(n-\tau_{11}\right)-a_{12}(n) x_{2}\left(n-\tau_{12}\right)-c_{1}(n)\right\}, \\
& x_{2}(n+1)=x_{2}(n) \exp \left\{\frac{a_{20}(n)}{x_{2}\left(n-\tau_{20}\right)+k_{2}(n)}-a_{21}(n) x_{1}\left(n-\tau_{21}\right)-a_{22}(n) x_{2}\left(n-\tau_{22}\right)-c_{2}(n)\right\},
\end{aligned}
$$

where $\left\{k_{i}(n)\right\},\left\{a_{i j}(n)\right\}$, and $\left\{c_{i}(n)\right\}$ are real positive bounded sequences, $\tau_{i j}(i=1,2 ; j=$ $0,1,2)$ are positive integers, $\tau=\max _{0 \leq i, j \leq 2}\left\{\tau_{i j}\right\}, x_{i}(s)=\phi_{i}(s) \geq 0, s \in[-\tau, 0] \cap Z, \phi_{i}(0)>0$, $i=1,2$.

On the other hand, as was pointed out by Huo and Li [14], ecosystem in the real world is continuously disturbed by unpredictable forces which can result in changes in the biological parameters such as survival rates. Practical interest in ecology is the question of whether or not an ecosystem can withstand those unpredictable disturbances which persist for a finite period of time. In the language of control variables, we call the disturbance functions as control variables. During the last decade, many scholars did excellent works on the feedback control ecosystems (see [14-21] and the references cited therein); however, most of those works are concerned with the continuous model and seldom did scholars considered the discrete ecosystem with feedback controls ([13, 15, 21]).

Recently, Li and Yang [15] proposed the following discrete $n$-species Schoener competition system with time delays and feedback controls:

$$
\begin{gathered}
x_{i}(k+1)=x_{i}(k) \exp \left\{\frac{r_{i}(k)}{x_{i}\left(k-\tau_{i}\right)+a_{i}(k)}-\sum_{j=1}^{n} b_{i j}(k) x_{j}\left(k-\tau_{j}\right)\right. \\
\left.-c_{i}(k)-d_{i}(k) u_{i}(k)-e_{i}(k) u_{i}\left(k-\eta_{i}\right)\right\}, \\
\Delta u_{i}(k)=-\alpha_{i}(k) u_{i}(k)+\beta_{i}(k) x_{i}(k)+\gamma_{i}(k) x_{i}\left(k-\sigma_{i}\right),
\end{gathered}
$$

where $x_{i}(k)(i=1,2, \ldots, n)$ is the density of competitive species at $k$ th generations; $u_{i}(k)$ is the control variable; $\Delta$ is the first-order forward difference operator $\Delta u_{i}(k)=u_{i}(k+1)-u_{i}(k)$, $i=1,2, \ldots, n$. 
Throughout this paper, we assume the following.

$\left(H_{1}\right) \alpha_{i}(k), \beta_{i}(k), \gamma_{i}(k), a_{i}(k), b_{i j}(k), r_{i}(k), c_{i}(k), d_{i}(k), e_{i}(k), i=1,2, \ldots, n$, are all bounded nonnegative sequences such that

$$
\begin{gathered}
0<\alpha_{i}^{l} \leq \alpha_{i}^{u}<1, \quad 0<\beta_{i}^{l} \leq \beta_{i}^{u}, \quad 0<r_{i}^{l} \leq r_{i}^{u}, \quad 0<a_{i}^{l} \leq a_{i}^{u}, \\
0<b_{i j}^{l} \leq b_{i j}^{u} \quad 0<r_{i}^{l} \leq r_{i}^{u}, \quad 0<c_{i}^{l} \leq c_{i}^{u}, \quad 0<d_{i}^{l} \leq d_{i}^{u}, \quad 0<e_{i}^{l} \leq e_{i}^{u} .
\end{gathered}
$$

Here, for any bounded sequence $\{a(k)\}, a^{u}=\sup _{k \in N} a(k), a^{l}=\inf _{k \in N} a(k)$.

$\left(H_{2}\right) \tau_{i}, \eta_{i}, \sigma_{i}, i=1,2, \ldots, n$, are all nonnegative integers.

Let $\tau=\max \left\{\tau_{i}, \eta_{i}, \sigma_{i}, i=1,2, \ldots, n\right\}$; we consider (1.3) together with the following initial conditions:

$$
\begin{array}{ll}
x_{i}(\theta)=\varphi_{i}(\theta), & \theta \in N[-\tau, 0]=\{-\tau,-\tau+1, \ldots, 0\}, \varphi_{i}(0)>0, \\
u_{i}(\theta)=\phi_{i}(\theta), & \theta \in N[-\tau, 0]=\{-\tau,-\tau+1, \ldots, 0\}, \phi_{i}(0)>0 .
\end{array}
$$

It is not difficult to see that solutions of (1.3) and (1.5) are well defined for all $k \geq 0$ and satisfy

$$
x_{i}(k)>0, \quad u_{i}(k)>0, \quad k \in Z, i=1,2, \ldots, n .
$$

By applying the comparison theorem of difference equation, they obtained a set of sufficient conditions which guarantee the permanence of the system (1.3). Their result shows that feedback control variables play important roles on the persistent property of the system (1.3). But the question is whether or not the feedback control variables have influence on the permanence of the system. The aim of this paper is to apply the analysis technique of Chen et al. [18] to establish sufficient conditions, which is independent of feedback control variables, to ensure the permanence of the system.

The organization of this paper is as follow. In Section 2, we will introduce several lemmas. The permanence of system (1.3) is then studied in Section 3. In Section 4, a suitable example together with its numerical simulations shows the feasibility of our results.

\section{Preliminaries}

In this section, we will introduce several useful lemmas.

Lemma 2.1 (see [11]). Assume that $\{x(k)\}$ satisfies $x(k)>0$ and

$$
x(k+1) \leq x(k) \exp \{a(k)-b(k) x(k)\}
$$

for $k \in N$, where $a(k)$ and $b(k)$ are nonnegative sequences bounded above and below by positive constants. Then

$$
\limsup _{k \rightarrow+\infty} x(k) \leq \frac{1}{b^{l}} \exp \left(a^{u}-1\right)
$$


Lemma 2.2 (see [12]). Assume that $\{x(k)\}$ satisfies

$$
x(k+1) \geq x(k) \exp \{a(k)-b(k) x(k)\}, \quad k \geq N_{0},
$$

$\lim \sup _{k \rightarrow+\infty} x(k) \leq x^{*}$ and $x\left(N_{0}\right)>0$, where $a(k)$ and $b(k)$ are nonnegative sequences bounded above and below by positive constants and $N_{0} \in N$. Then

$$
\liminf _{k \rightarrow+\infty} x(k) \geq \min \left\{\frac{a^{l}}{b^{u}} \exp \left(a^{l}-b^{u} x^{*}\right), \frac{a^{l}}{b^{u}}\right\} .
$$

Lemma 2.3 (see [13]). Assume that $A>0, y(0)>0$. Suppose that

$$
y(k+1) \leq A y(k)+B(k), \quad k=1,2, \ldots
$$

Then for any integer $m \leq k$,

$$
y(k) \leq A^{m} y(k-m)+\sum_{j=0}^{m-1} A^{j} B(k-j-1) .
$$

If $A<1$ and $B$ is bounded above with respect to $M$, then

$$
\limsup _{k \rightarrow+\infty} y(k) \leq \frac{M}{1-A}
$$

Lemma 2.4 (see [13]). Assume that $A>0, y(0)>0$. Suppose that

$$
y(k+1) \geq A y(k)+B(k), \quad k=1,2, \ldots
$$

Then for any integer $m \leq k$,

$$
y(k) \geq A^{m} y(k-m)+\sum_{j=0}^{m-1} A^{j} B(k-j-1) .
$$

If $A<1$ and $B$ is bounded below with respect to $P$, then

$$
\liminf _{k \rightarrow+\infty} y(k) \geq \frac{P}{1-A} .
$$




\section{Permanence}

In this section, we establish the following permanence result for system (1.3).

Theorem 3.1. Assume that $\left(H_{1}\right)$ and $\left(H_{2}\right)$ hold. Then there exist positive constants $M_{i}, N_{i}$ which are independent of the solutions of system (1.3) such that

$$
\begin{gathered}
\limsup _{k \rightarrow \infty} x_{i}(k) \leq M_{i}, \quad i=1,2, \ldots, n, \\
\limsup _{k \rightarrow \infty} u_{i}(k) \leq N_{i}, \quad i=1,2, \ldots, n .
\end{gathered}
$$

Proof. Let $x(k)=\left(x_{1}(k), x_{2}(k) \cdots x_{n}(k), u_{1}(k), u_{2}(k) \cdots u_{n}(k)\right)^{T}$ be any positive solution of system (1.3) with initial condition (1.5). From the first equation of system (1.3), it follows that

$$
x_{i}(k+1) \leq x_{i}(k) \exp \left\{\frac{r_{i}(k)}{a_{i}(k)}-b_{i i}(k) x_{i}\left(k-\tau_{i}\right)\right\} \leq x_{i}(k) \exp \left\{\frac{r_{i}^{u}}{a_{i}^{l}}\right\}
$$

By using (3.2), one can easily obtain that

$$
\prod_{j=k-\tau_{i}}^{k-1} x_{i}(j+1) \leq \prod_{j=k-\tau_{i}}^{k-1} x_{i}(j) \exp \left\{\frac{r_{i}^{u}}{a_{i}^{l}}\right\}
$$

that is,

$$
x_{i}(k) \leq x_{i}\left(k-\tau_{i}\right) \exp \left\{\frac{\tau_{i} r_{i}^{u}}{a_{i}^{l}}\right\}
$$

Substituting (3.4) into (3.2), it follows that

$$
x_{i}(k+1) \leq x_{i}(k) \exp \left\{\frac{r_{i}(k)}{a_{i}(k)}-b_{i i}(k) \exp \left\{-\frac{\tau_{i} r_{i}^{u}}{a_{i}^{l}}\right\} x_{i}(k)\right\} .
$$

By applying Lemma 2.1, it follows that

$$
\limsup _{k \rightarrow \infty} x_{i}(k) \leq \frac{\exp \left\{\left(r_{i}^{u} / a_{i}^{l}\right)\left(1+\tau_{i}\right)-1\right\}}{b_{i i}^{l}}=: M_{i}, \quad i=1,2, \ldots, n .
$$


Thus, for all $\varepsilon>0$, there exists a $K_{1}>0, K_{1} \in N$, for all $k>K_{1}, x_{i}(k) \leq M_{i}+\varepsilon, i=1,2, \ldots, n$, and so

$$
\begin{aligned}
u_{i}(k+1) & =\left(1-\alpha_{i}(k)\right) u_{i}(k)+\beta_{i}(k) x_{i}(k)+\gamma_{i}(k) x_{i}\left(k-\sigma_{i}\right) \\
& \leq\left(1-\alpha_{i}^{l}\right) u_{i}(k)+\left(\beta_{i}^{u}+\gamma_{i}^{u}\right)\left(M_{i}+\varepsilon\right) \quad\left(k>K_{1}+\sigma_{i}\right) .
\end{aligned}
$$

For $0<1-\alpha_{i}^{l}<1$, Lemma 2.3 implies that

$$
\limsup _{k \rightarrow \infty} u_{i}(k) \leq \frac{\left(\beta_{i}^{u}+\gamma_{i}^{u}\right)\left(M_{i}+\varepsilon\right)}{\alpha_{i}^{l}}
$$

Letting $\varepsilon \rightarrow 0$ in the above inequality leads to

$$
\limsup _{k \rightarrow \infty} u_{i}(k) \leq \frac{\left(\beta_{i}^{u}+\gamma_{i}^{u}\right) M_{i}}{\alpha_{i}^{l}}:=N_{i}
$$

The proof of Theorem 3.1 is completed.

Theorem 3.2. Assume that $\left(H_{1}\right)$ and $\left(H_{2}\right)$ hold; assume further that

$$
R_{i}:=\frac{r_{i}^{l}}{M_{i}+a_{i}^{u}}-\sum_{j=1, j \neq i}^{n} b_{i j}^{u} M_{j}-c_{i}^{u}>0, \quad i=1,2, \ldots, n
$$

Then there exist positive constants $m_{i}, n_{i}$ which are independent of the solution of system (1.3), such that

$$
\begin{aligned}
& \liminf _{k \rightarrow \infty} x_{i}(k) \geq m_{i}, \quad i=1,2, \ldots, n \\
& \liminf _{k \rightarrow \infty} u_{i}(k) \geq n_{i}, \quad i=1,2, \ldots, n .
\end{aligned}
$$

Proof. Let $x(k)=\left(x_{1}(k), x_{2}(k) \cdots x_{n}(k), u_{1}(k), u_{2}(k) \cdots u_{n}(k)\right)^{T}$ be any positive solution of system (1.3) with initial condition (1.5). From Theorem 3.1, for all $0<\varepsilon<1$, there exists a $K_{2}>K_{1}$, for all $k>K_{2}$,

$$
\begin{gathered}
x_{i}(k) \leq M_{i}+\varepsilon, \quad u_{i}(k) \leq N_{i}+\varepsilon, \quad i=1,2, \ldots, n, \\
R_{i \varepsilon}:=\frac{r_{i}^{l}}{\left(M_{j}+\varepsilon\right)+a_{i}^{u}}-\sum_{j=1, j \neq i}^{n} b_{i j}^{u}\left(M_{j}+\varepsilon\right)-c_{i}^{u}>0, \quad i=1,2, \ldots, n .
\end{gathered}
$$


From (3.12), we have

$$
\begin{aligned}
x_{i}(k+1) \geq x_{i}(k) \exp \left\{\frac{r_{i}^{l}}{\left(M_{i}+\varepsilon\right)+a_{i}^{u}}-\sum_{j=1, j \neq i}^{n} b_{i j}^{u}\left(M_{j}+\varepsilon\right)\right. \\
\left.\quad-c_{i}^{u}-d_{i}^{u} u_{i}(k)-e_{i}^{u} u_{i}\left(k-\eta_{i}\right)-b_{i i}^{u} x_{i}\left(k-\tau_{i}\right)\right\} \\
\geq x_{i}(k) \exp \left\{-\sum_{j=1}^{n} b_{i j}^{u}\left(M_{j}+\varepsilon\right)-c_{i}^{u}-\left(d_{i}^{u}+e_{i}^{u}\right)\left(N_{i}+\varepsilon\right)\right\} \\
=: x_{i}(k) \exp \left\{D_{i \varepsilon}\right\} .
\end{aligned}
$$

for all $k>K_{2}+\tau$, where $D_{i \varepsilon}=-\sum_{j=1}^{n} b_{i j}^{u}\left(M_{j}+\varepsilon\right)-c_{i}^{u}-\left(d_{i}^{u}+e_{i}^{u}\right)\left(N_{i}+\varepsilon\right)<0$.

Thus, for $\eta<K$, by using (3.13) we obtain

$$
x_{i}(\eta) \leq x_{i}(k) \exp \left\{-(k-\eta) D_{i \varepsilon}\right\}
$$

From the second equation of (1.3), we obtain

$$
\begin{aligned}
u_{i}(k+1) & =\left(1-\alpha_{i}(k)\right) u_{i}(k)+\beta_{i}(k) x_{i}(k)+\gamma_{i}(k) x_{i}\left(k-\sigma_{i}\right) \\
& \leq\left(1-\alpha_{i}^{l}\right) u_{i}(k)+\beta_{i}^{u} x_{i}(k)+\gamma_{i}^{u} x_{i}\left(k-\sigma_{i}\right) \\
& \triangleq A_{i} u_{i}(k)+B_{i}(k)
\end{aligned}
$$

where $0<A_{i}=1-\alpha_{i}^{l}<1$ and $B_{i}(k)=\beta_{i}^{u} x_{i}(k)+\gamma_{i}^{u} x_{i}\left(k-\sigma_{i}\right)$.

Then, Lemma 2.3, (3.14), and (3.15) imply that for any $m \leq K$,

$$
\begin{aligned}
u_{i}(k) \leq & A_{i}^{m} u_{i}(k-m)+\sum_{j=0}^{m-1} A_{i}^{j} B_{i}(k-j-1) \\
= & A_{i}^{m} u_{i}(k-m)+\sum_{j=0}^{m-1} A_{i}^{j}\left\{\beta_{i}^{u} x_{i}(k-j-1)+\gamma_{i}^{u} x_{i}\left(k-j-1-\sigma_{i}\right)\right\} \\
\leq & A_{i}^{m} u_{i}(k-m) \\
& +\sum_{j=0}^{m-1} A_{i}^{j}\left\{\beta_{i}^{u} \exp \left\{-(j+1) D_{i \varepsilon}\right\}+\gamma_{i}^{u} \exp \left\{-\left(j+1+\sigma_{i}\right) D_{i \varepsilon}\right\}\right\} x_{i}(k) \\
\leq & A_{i}^{m} u_{i}(k-m)+x_{i}(k) \sum_{j=0}^{m-1} A_{i}^{j}\left(\beta_{i}^{u}+\gamma_{i}^{u}\right) \exp \left\{-(j+1) D_{i \varepsilon}\right\} .
\end{aligned}
$$


For $0<A_{i}<1, \lim _{m \rightarrow \infty}\left(d_{i}^{u}+e_{i}^{u}\right)\left(N_{i}+1\right) A_{i}^{m}=0$. That is, there exists an $M>0$, for all $m>M$

$$
\left(N_{i}+\varepsilon\right)\left(d_{i}^{u}+e_{i}^{u}\right) A_{i}^{m}<\left(N_{i}+1\right)\left(d_{i}^{u}+e_{i}^{u}\right) A_{i}^{m} \leq \frac{R_{i \varepsilon_{0}}}{2} \leq \frac{R_{i \varepsilon}}{2} .
$$

For enough small $\varepsilon_{0}>0$, we have

$$
R_{i \varepsilon_{0}}:=\frac{r_{i}^{l}}{\left(M_{i}+\varepsilon_{0}\right)+a_{i}^{u}}-\sum_{j=1, j \neq i}^{n} b_{i j}^{u}\left(M_{j}+\varepsilon_{0}\right)-c_{i}^{u}<R_{i \varepsilon}<R_{i}, \quad i=1,2, \ldots, n .
$$

We choose $M \geq \max \left\{1, \log _{A_{i}}\left(R_{i \varepsilon_{0}} / 2\left(N_{i}+1\right)\left(d_{i}^{u}+e_{i}^{u}\right)\right)\right\}, i=1,2, \ldots, n$. For fixed $M$, we get

$$
\begin{aligned}
u_{i}(k) & \leq A_{i}^{M} u_{i}(k-M)+x_{i}(k) \sum_{j=0}^{M-1} A_{i}^{j}\left(\beta_{i}^{u}+\gamma_{i}^{u}\right) \exp \left\{-(j+1) D_{i \varepsilon}\right\} \\
& \leq A_{i}^{M}\left(N_{i}+\varepsilon\right)+E_{i \varepsilon} x_{i}(k)
\end{aligned}
$$

for all $k \geq K_{2}+M$ where $E_{i \varepsilon}=\sum_{j=0}^{M-1} A_{i}^{j}\left(\beta_{i}^{u}+\gamma_{i}^{u}\right) \exp \left\{-(j+1) D_{i \varepsilon}\right\}$.

Substituting (3.14) (3.17) and (3.19) into (3.13), for $k \geq K_{2}+M+\tau$, one has

$$
\begin{aligned}
x_{i}(k+1) & \geq x_{i}(k) \exp \left\{R_{i \varepsilon}-b_{i i}^{u} x_{i}\left(k-\tau_{i}\right)-d_{i}^{u} u_{i}(k)-e_{i}^{u} u_{i}\left(k-\eta_{i}\right)\right\} \\
& \geq x_{i}(k) \exp \left\{R_{i \varepsilon}-\left(e_{i}^{u}+d_{i}^{u}\right) A_{i}^{M}\left(N_{i}+\varepsilon\right)-b_{i i}^{u} x_{i}\left(k-\tau_{i}\right)-d_{i}^{u} E_{i \varepsilon} x_{i}(k)-e_{i}^{u} E_{i \varepsilon} x_{i}\left(k-\eta_{i}\right)\right\} \\
& \geq x_{i}(k) \exp \left[\frac{R_{i \varepsilon}}{2}-b_{i i}^{u} \exp \left\{-\tau_{i} D_{i \varepsilon}\right\}-d_{i}^{u} E_{i \varepsilon}-e_{i}^{u} E_{i \varepsilon} \exp \left\{-\eta_{i} D_{i \varepsilon}\right\}\right] x_{i}(k) \\
& :=x_{i}(k) \exp \left\{\frac{R_{i \varepsilon}}{2}-Q_{i \varepsilon} x_{i}(k)\right\}
\end{aligned}
$$

where $Q_{i \varepsilon}:=b_{i i}^{u} \exp \left\{-\tau_{i} D_{i \varepsilon}\right\}+d_{i}^{u} E_{i \varepsilon}+e_{i}^{u} E_{i \varepsilon} \exp \left\{-\eta_{i} D_{i \varepsilon}\right\}$.

By applying Lemma 2.2, it follows that

$$
\liminf _{k \rightarrow \infty} x(k) \geq \min \left\{\frac{R_{i \varepsilon}}{2 Q_{i \varepsilon}} \exp \left\{\frac{R_{i \varepsilon}}{2}-Q_{i \varepsilon} M_{i}\right\}, \frac{R_{i \varepsilon}}{2 Q_{i \varepsilon}}\right\} .
$$

Letting $\varepsilon \rightarrow 0$ in the above inequality leads to

$$
\liminf _{k \rightarrow \infty} x(k) \geq \min \left\{\frac{R_{i}}{2 Q_{i}} \exp \left\{\frac{R_{i}}{2}-Q_{i} M_{i}\right\}, \frac{R_{i}}{2 Q_{i}}\right\}:=m_{i} .
$$


For the above $\varepsilon>0$, there exists a $K_{3}>K_{2}$, for all $k>K_{3}$, we have $x_{i}(k) \geq m_{i}-\varepsilon, i=1,2, \ldots, n$. Then for $k>K_{3}+\sigma_{i}$

$$
\begin{aligned}
u_{i}(k+1) & =\left(1-\alpha_{i}(k)\right) u_{i}(k)+\beta_{i}(k) x_{i}(k)+\gamma_{i}(k) x_{i}\left(k-\sigma_{i}\right) \\
& \geq\left(1-\alpha_{i}^{u}\right) u_{i}(k)+\left(\beta_{i}^{l}+\gamma_{i}^{l}\right)\left(m_{i}-\varepsilon\right) .
\end{aligned}
$$

By applying Lemma 2.4, it follows that

$$
\liminf _{k \rightarrow \infty} u_{i}(k) \geq \frac{\left(\beta_{i}^{l}+\gamma_{i}^{l}\right)\left(m_{i}-\varepsilon\right)}{\alpha_{i}^{u}} .
$$

Letting $\varepsilon \rightarrow 0$ in the above inequality leads to

$$
\liminf _{k \rightarrow \infty} u_{i}(k) \geq \frac{\left(\beta_{i}^{l}+\gamma_{i}^{l}\right) m_{i}}{\alpha_{i}^{u}}:=n_{i}, \quad i=1,2, \ldots, n
$$

The proof of Theorem 3.2 is completed.

Remark 3.3. Theorems 3.1 and 3.2 show that under the assumption $\left(H_{1}\right),\left(H_{2}\right)$ and $(H)$ hold, and system (1.3) is permanent.

\section{Example and Numeric Simulations}

The following example lends credence to the plausibility of Theorem 3.2.

Example 4.1. Consider the following system:

$$
\begin{aligned}
& x_{1}(k+1)=x_{1}(k) \exp \left\{\frac{0.5}{x_{1}(k-1)+1}-x_{1}(k-1)-0.02(\sin (k)+2) x_{2}(k-2)\right. \\
& \left.-0.01-(\cos (k)+2.5) u_{1}(k)-(\cos (k)+1.5) u_{1}(k-1)\right\}, \\
& x_{2}(k+1)=x_{2}(k) \exp \left\{\frac{1}{x_{2}(k-2)+3}-0.05(\cos (k)+2) x_{1}(k-1)-2 x_{2}(k-2)\right. \\
& \left.-0.05-(\sin (k)+2) u_{2}(k)-(\sin (k)+1.1) u_{2}(k-1)\right\}, \\
& \Delta u_{1}(k)=-0.5 u_{1}(k)+0.1(\sin (10+k)+1.5) x_{1}(k)+0.1(\sin (k)+1.5) x_{1}(k-1), \\
& \Delta u_{2}(k)=-\frac{1}{3} u_{2}(k)+0.1(\cos (10+k)+1.5) x_{2}(k)+0.1(\cos (k+5)+1.5) x_{2}(k-1) \text {. }
\end{aligned}
$$




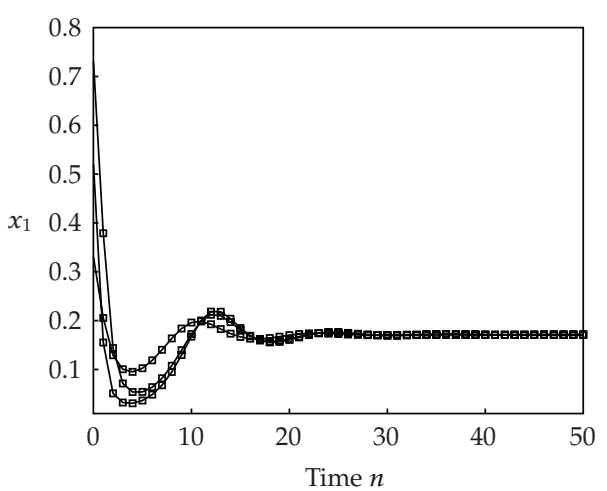

$\rightarrow x_{1}$

(a)

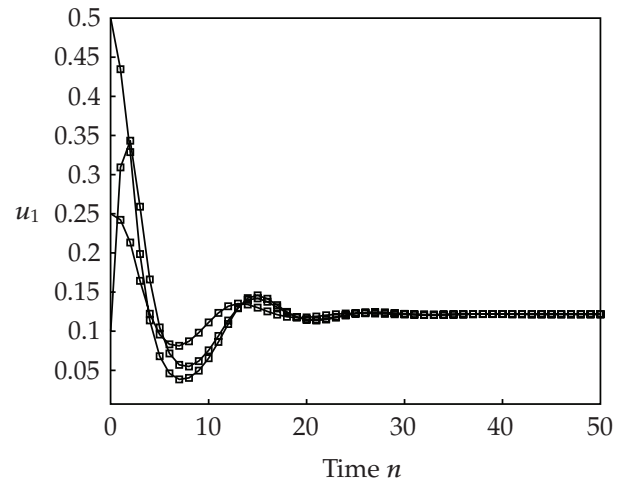

$\rightarrow u_{1}$

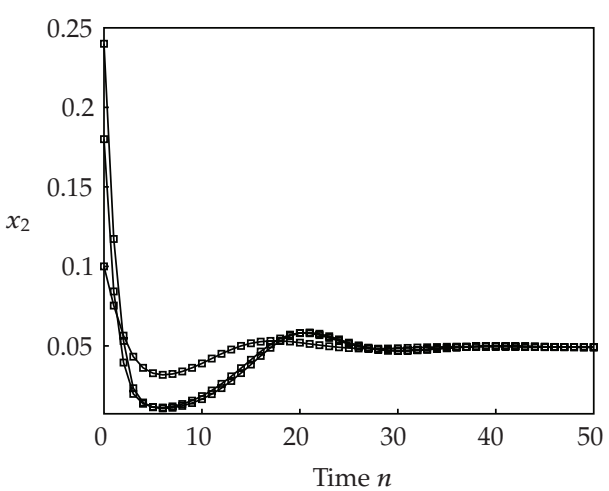

$\rightarrow x_{2}$

(b)

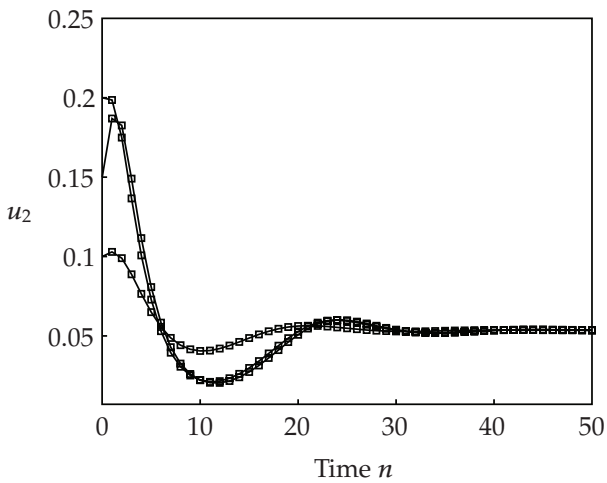

$\square-u_{2}$

(c)

(d)

Figure 1: Dynamic behaviors of system (4.1) with initial conditions $\left(x_{1}(s), x_{2}(s), u_{1}(s), u_{2}(s)\right)^{T}=$ $(0.73,0.1,0.1,0.1)^{T},(0.33,0.24,0.25,0.15)^{T},(0.52,0.18,0.5,0.2)^{T}, s=-1,0$.

Here corresponding to system (1.3), we assume that

$$
\begin{gathered}
r_{1}(k)=0.5, \quad a_{1}(k)=1, \quad b_{11}(k)=1, \quad b_{12}(k)=0.02(\sin (k)+2), \\
c_{1}(k)=0.01, \quad \eta_{1}=1, \quad d_{1}(k)=\cos (k)+2.5, \quad e_{1}(k)=\cos (k)+1.5, \\
\alpha_{1}(k)=0.5, \quad \beta_{1}(k)=0.1(\sin (k+10)+1.5), \\
r_{1}(k)=0.1(\sin (k)+1.5), \quad \sigma_{1}=1, \\
r_{2}(k)=1, \quad \tau_{2}=2, \quad a_{2}(k)=3, \quad b_{22}(k)=2, \quad b_{21}(k)=0.05(\cos (k)+2), \\
c_{2}(k)=0.05, \quad \eta_{2}=1, \quad d_{2}(k)=\sin (k)+2, \quad e_{2}(k)=\sin (k)+1.1, \\
\alpha_{2}(k)=\frac{1}{3}, \quad \beta_{2}(k)=0.1(\cos (k+10)+1.5), \\
r_{2}(k)=0.1(\cos (k+5)+1.5), \quad \sigma_{2}=1 .
\end{gathered}
$$


It is easy to see that $M_{1}=1, M_{2}=0.5$, and

$$
\begin{aligned}
& \frac{r_{1}^{l}}{M_{1}+a_{1}^{u}}-b_{12}^{u} M_{2}-c_{1}^{u}=0.21>0, \\
& \frac{r_{2}^{l}}{M_{2}+a_{2}^{u}}-b_{21}^{u} M_{1}-c_{2}^{u}=\frac{3}{35}>0 .
\end{aligned}
$$

Inequalities (4.3) show that all the conditions of Theorem 3.2 are satisfied; thus, system (1.3) is permanent. Numerical simulation from Figure 1 supports this conclusion.

\section{Discussion}

In this paper we have attempted to understand the effect of feedback control variables on the permanence of system (1.3). The present work is an extension of an earlier work by Li and Yang [15]. By developing the analysis technique of Chen et al. [18], a set of sufficient conditions are established for the permanence of system (1.3). Theorems 3.1-3.2 and the numerical simulations indicate that feedback control variables have no influence on the permanence of system (1.3).

We would like to mention here that an interesting but challenging problem associated with the study of system (1.3) should be the global attractivity. We leave this for future work.

\section{Acknowledgments}

The authors would like to thank the two anonymous referees for their constructive suggestions on improving the presentation of the paper. Also, this work is supported by the Technology Innovation Platform Project of Fujian Province (2009J1007).

\section{References}

[1] Q. M. Liu, R. Xu, and W. G. Wang, "Global asymptotic stability of Schoener's competitive model with delays," Journal of Biomathematics, vol. 21, no. 1, pp. 147-152, 2006.

[2] G.-L. Chen, R. Xu, and Q.-M. Liu, "Periodic solutions of a competitive model with distributed time delays," Journal of Biomathematics, vol. 19, no. 4, pp. 385-394, 2004 (Chinese).

[3] Z. H. Lu and L. S. Chen, "Analysis on a periodic Schoener model," Acata Mathematica Scientia, vol. 12, no. 7, supplement, pp. 105-109, 1992.

[4] H. Xiang, K. M. Yan, and B. Y. Wang, "Positive periodic solutions for discrete Schoner competitive models," Journal of Lanzhou University of Technology, vol. 31, no. 5, pp. 125-128, 2005 (Chinese).

[5] L. Wu, F. Chen, and Z. Li, "Permanence and global attractivity of a discrete Schoener's competition model with delays," Mathematical and Computer Modelling, vol. 49, no. 7-8, pp. 1607-1617, 2009.

[6] Z. Li and F. Chen, "Extinction in two dimensional discrete Lotka-Volterra competitive system with the effect of toxic substances," Dynamics of Continuous, Discrete E Impulsive Systems. Series B, vol. 15, no. 2, pp. 165-178, 2008.

[7] Y. Chen and Z. Zhou, "Stable periodic solution of a discrete periodic Lotka-Volterra competition system," Journal of Mathematical Analysis and Applications, vol. 277, no. 1, pp. 358-366, 2003.

[8] Z. Zhou and X. Zou, "Stable periodic solutions in a discrete periodic logistic equation," Applied Mathematics Letters, vol. 16, no. 2, pp. 165-171, 2003.

[9] Y. Muroya, "Persistence and global stability in discrete models of Lotka-Volterra type," Journal of Mathematical Analysis and Applications, vol. 330, no. 1, pp. 24-33, 2007. 
[10] W. Wang, G. Mulone, F. Salemi, and V. Salone, "Global stability of discrete population models with time delays and fluctuating environment," Journal of Mathematical Analysis and Applications, vol. 264, no. 1, pp. 147-167, 2001.

[11] F. Chen, "Permanence and global attractivity of a discrete multispecies Lotka-Volterra competition predator-prey systems," Applied Mathematics and Computation, vol. 182, no. 1, pp. 3-12, 2006.

[12] F. Chen, "Permanence for the discrete mutualism model with time delays," Mathematical and Computer Modelling, vol. 47, no. 3-4, pp. 431-435, 2008.

[13] Y.-H. Fan and L.-L. Wang, "Permanence for a discrete model with feedback control and delay," Discrete Dynamics in Nature and Society, vol. 2008, Article ID 945109, 8 pages, 2008.

[14] H.-F. Huo and W.-T. Li, "Positive periodic solutions of a class of delay differential system with feedback control," Applied Mathematics and Computation, vol. 148, no. 1, pp. 35-46, 2004.

[15] X. Li and W. Yang, "Permanence of a discrete $n$-species Schoener competition system with time delays and feedback controls," Advances in Difference Equations, vol. 2009, Article ID 515706, 10 pages, 2009.

[16] F. Chen, "Permanence in nonautonomous multi-species predator-prey system with feedback controls," Applied Mathematics and Computation, vol. 173, no. 2, pp. 694-709, 2006.

[17] F. Chen, "Permanence of a discrete $N$-species cooperation system with time delays and feedback controls," Applied Mathematics and Computation, vol. 186, no. 1, pp. 23-29, 2007.

[18] L. Chen, L. Chen, and Z. Li, "Permanence of a delayed discrete mutualism model with feedback controls," Mathematical and Computer Modelling, vol. 50, no. 7-8, pp. 1083-1089, 2009.

[19] F. Chen, "Positive periodic solutions of neutral Lotka-Volterra system with feedback control," Applied Mathematics and Computation, vol. 162, no. 3, pp. 1279-1302, 2005.

[20] F. Chen, X. Liao, and Z. Huang, "The dynamic behavior of N-species cooperation system with continuous time delays and feedback controls," Applied Mathematics and Computation, vol. 181, no. 2, pp. 803-815, 2006.

[21] L. Chen, X. Xie, and L. Chen, "Feedback control variables have no influence on the permanence of a discrete $N$-species cooperation system," Discrete Dynamics in Nature and Society, vol. 2009, Article ID 306425, 10 pages, 2009. 


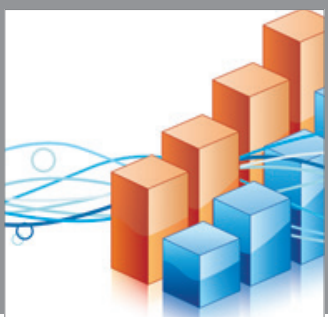

Advances in

Operations Research

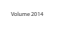

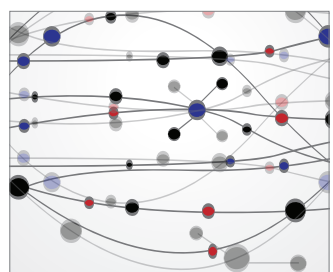

\section{The Scientific} World Journal
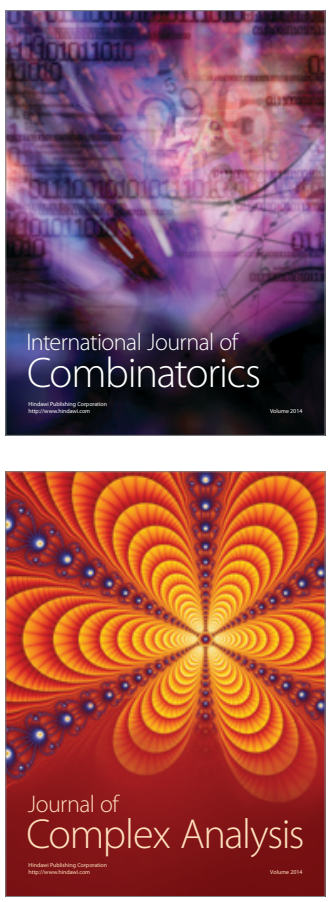

International Journal of

Mathematics and

Mathematical

Sciences
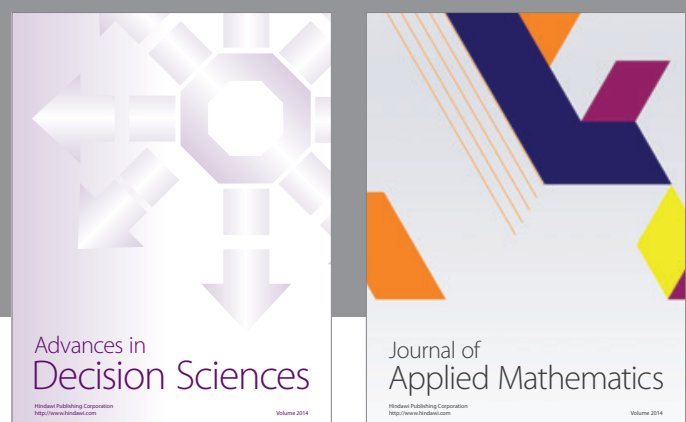

Journal of

Applied Mathematics
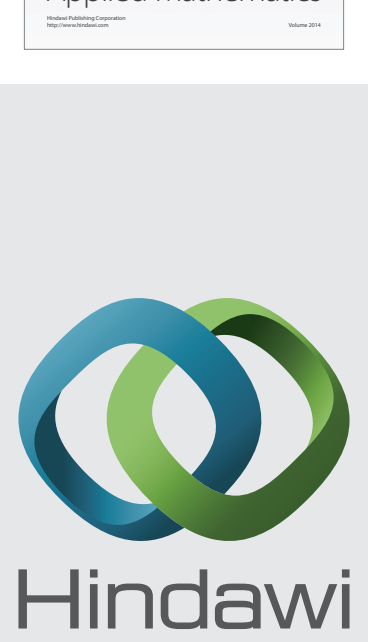

Submit your manuscripts at http://www.hindawi.com
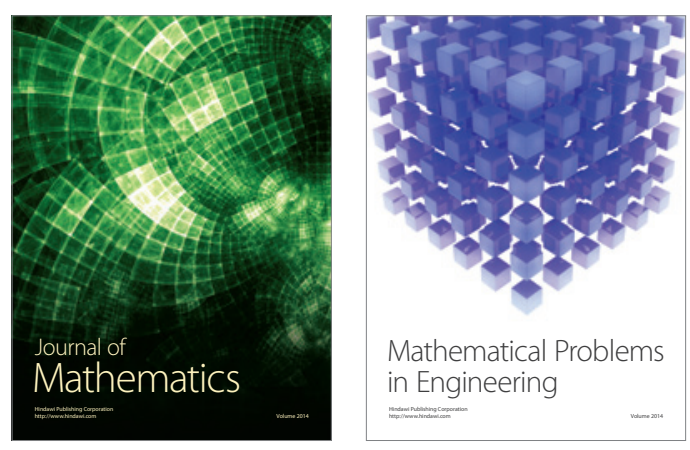

Mathematical Problems in Engineering
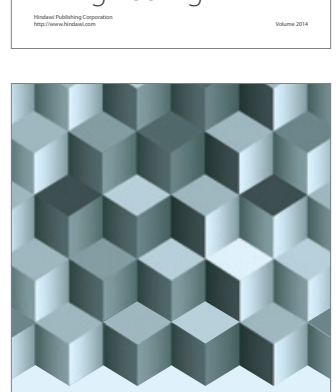

Journal of

Function Spaces
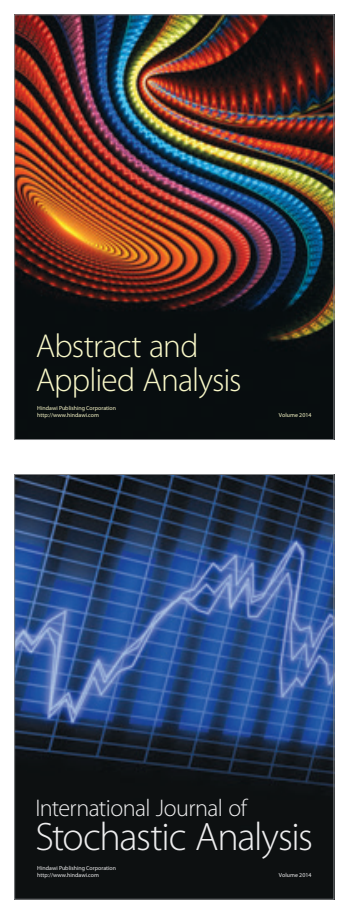

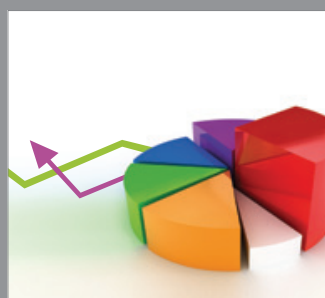

ournal of

Probability and Statistics

Promensencen
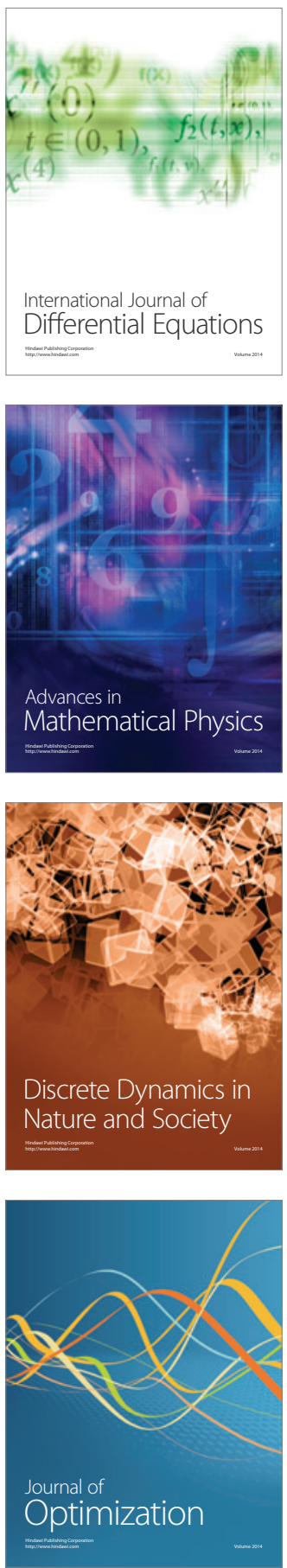